\title{
Nighttime gibberellin biosynthesis is influenced by fluctuating environmental conditions and contributes to growth adjustments of Arabidopsis leaves.
}

Putri Prasetyaningrum ${ }^{1}$, Lorenzo Mariotti ${ }^{2}$, Maria Cristina Valeri ${ }^{1}$, Giacomo Novi ${ }^{1}$, Stijn Dhondt ${ }^{3,4}$, Dirk Inzé ${ }^{3,4}$, Pierdomenico Perata ${ }^{1 *}$, Hans van Veen ${ }^{1,5^{*}}$

1 PlantLab, Institute of Life Sciences, Scuola Superiore Sant'Anna, Pisa 56127, Italy

2 Dept. of Agriculture, Food and Environment, University of Pisa, Pisa 56124, Italy

3 Center for Plant Systems Biology, VIB, 9052 Ghent, Belgium

4 Department of Plant Biotechnology and Bioinformatics, Ghent University, 9052 Ghent, Belgium

5 Plantecophysiology, Institute of Environmental Biology, Utrecht University, $3584 \mathrm{CH}$ Utrecht

*Corresponding authors, p.perata@santannapisa.it, h.vanveen@uu.nl

Author contributions: P.Pe and H.v.V conceived the project; P.Pr, S.D., D.I. P.Pe and H.v.V designed experiments; P.Pr, L.M., M.C.V., G.N. and H.v.V Performed Experiments; P.Pr, S.D. and H.v.V analysed the data; H.v.V. and P.Pe wrote the paper with contributions from S.D. D.I. 


\begin{abstract}
Optimal plant growth performance requires that the action of growth signals, such as gibberellins (GA), are coordinated with the availability of photo-assimilates. Here, we studied the links between gibberellin biosynthesis and carbon availability, and the subsequent effects on growth. The results presented here show that carbon availability, light and dark cues, and the clock ensure the timing and magnitude of gibberellin biosynthesis and that disruption of these mechanisms results in reduced gibberellin levels and expression of downstream genes. Carbon dependent nighttime induction of GIBBERELLIN 3-BETA-DIOXYGENASE 1 (GA3OX1) was severely hampered when preceded by a day of lowered light availability, leading specifically to reduced bioactive $\mathrm{GA}_{4}$ levels, and coinciding with a decline in leaf expansion rate during the night. We attribute this decline in leaf expansion mostly to reduced photo-assimilates. However, plants where gibberellin limitation was alleviated had significantly improved expansion demonstrating the relevance of gibberellins in growth control under varying carbon availability. Carbon dependent expression of upstream gibberellin biosynthesis genes (KAURENE SYNTHASE, KS and GIBBERELLIN 20 OXIDASE 1, GA20ox1) was not translated into metabolite changes with in this short timeframe. We propose a model where the extent of nighttime biosynthesis of bioactive $\mathrm{GA}_{4}$ by GA3ox1 is determined by starch, as the nighttime carbon source, and so provides day-to-day adjustment of gibberellin responses.
\end{abstract}

\title{
Introduction
}

Growth in plants is controlled by many signals, consisting of both environmental and internal cues. Growth is a complex parameter often interpreted as the increase in the number and size of organs (1). Others consider growth the gain of biomass, sometimes more specifically as incorporation of carbon into structural carbohydrates (2-3). Growth parameters like protein and cell wall synthesis directly follow carbon availability (4-5). Carbon storage and utilization are organized such that carbohydrates are roughly evenly incorporated into structural biomass throughout the day (2-3). However, size increases of plants do not necessarily follow carbon availability. Hypocotyl and leaf expansion rates have been shown to vary throughout the day, and these expansion patterns have been attributed to the circadian clock and light signalling (6-8). Interestingly, expansion and metabolic biosynthesis can be distinctly regulated. In hypocotyls, cell wall biosynthesis was exclusively depended on metabolic signals and cell expansion on the circadian clock (5). For optimal plant performance, size increase must match biomass integration. The ability to adjust leaf expansion rates based on resource availability would be especially important when conditions vary from day to day.

In conjunction with the clock and light signals, a variety of growth stimulating signalling networks exists within the plant, including a set that rely on phytohormones (1). Pivotal among these are gibberellins (GAs), which positively regulate cell expansion and cell division (9-10). Plants deficient in gibberellins develop slowly and are typically very small in stature (11), whereas overexpression of biosynthetic routes or gibberellin signalling leads to enhanced plant size (12-15). Gibberellin biosynthesis occurs via a sequence of enzymatic steps, starting in the plastids and subsequently the endoplasmic reticulum, which convert geranylgeranyl diphosphate to $G_{12}$. $G A_{12}$ is considered the common precursor to all gibberellins in plants and is further processed into a variety of forms by GIBBERELLIN 20-OXIDASEs (GA20ox). The final step to bioactive gibberellin is catalysed by GIBBERELLIN 3-BETADIOXYGENASE (GA3ox) (11). In higher plants $\mathrm{GA}_{4}, \mathrm{GA}_{1}$ and $\mathrm{GA}_{3}$ have been confirmed to bind the GIBBERELLIN INSENSTIVE DWARF1 (GID1) receptor (16-17). These bioactive gibberellins can also be made inactive by the enzyme GA2ox (11).

Established aspects of the regulation of gibberellin biosynthesis concern localized suppression in the shoot apical meristem to ensure stem cell maintenance, and feedback regulation that is considered important to maintain gibberellin homeostasis (18-19). Feedback regulation is structured such that it aims to keep gibberellin levels constant (19) and is therefore not adequate to explain growth adjustments to varying conditions. To identify the role of gibberellins in vegetative growth and adjustment to adverse conditions we studied the regulation of key gibberellin biosynthetic genes, the subsequent variation in gibberellin abundance and ultimately their role in 
growth in Arabidopsis thaliana. This led to the finding that nighttime conversion of precursors to bioactive $\mathrm{GA}_{4}$ by the starvation sensitive GA3ox1, plays a role in adjusting growth to adverse conditions.

\section{Results}

\section{Light, the circadian clock and carbon availability regulate the timing and transcriptional induction of GA biosynthetic enzymes}

Several enzymatic steps of GA biosynthesis are encoded by multigene families. To identify the key family members with relatively high expression during vegetative growth, two public microarray datasets that followed transcript abundance throughout 48 hours in adult Arabidopsis rosettes were investigated (20-21). Further indication of a role in adjusting growth control was based on oscillatory behaviour over the day-night cycle, indicating a responsiveness to changes in carbon supply, light signals or the clock. These data (Suppl. Figure 1) pointed to KAURENE SYNTHASE (KS), GA20ox1 and GA3ox1 as main candidates (Figure 1).

Clock components and clock-regulated genes retain their oscillations after a transfer to continuous light conditions (Supplementary Figure S2A). Of the daytime expressed candidates, oscillations were retained for $K S$, but not for GA20ox1. However, their peak in expression during normal day/night cycles, start of the day (GA20ox1) and afternoon (KS), matched the publicly available dataset (Figure 2A). GA20ox1 oscillations seemed independent of the clock (Figure $2 \mathrm{~A}$ ), and a role for light in inducing this gene was investigated by exposing plants to light three hours prior to expected dawn. An earlier start of the day resulted in a concomitant earlier peak of GA20ox1 (Figure 2B). Since an early day also led to a sooner increase in sugars (Figure 2B, Supplementary Figure S2B), a role for photosynthates to induce the earlier peak could not be excluded. After inhibiting photosynthesis with DCMU, GA20ox1 still peaked upon earlier light exposure. However, DCMU did reduce the extent of induction and abolished GA20ox1 induction at later timepoints (Figure 2B). Carbon starvation reduced GA20ox1 expression, but a DCMU treatment can be very severe, as apparent from the very strong induction of carbon starvation marker genes, DIN6 and TPS8 (Supplemental Figure S2B). Therefore, plants were also exposed to low light conditions that were sufficient to maintain a positive carbon balance but did reduce photosynthesis and also showed minor induction of starvation markers compared to the DCMU treatment (Supplementary Figure S2C-E). Both KS and GA20ox1 showed severely reduced daytime expression after DCMU treatment, whereas the low light treatment led, as expected, to an intermediate reduction in expression levels (Figure 2C).

Regarding the nighttime expressed candidate, GA3ox1 induction was induced upon transition to darkness, in line with public data (Figure 1 and $3 A$ ). Oscillations of GA3ox1 were not retained under continuous light, indicating that a shift to darkness is essential for a GA3ox1 induction (Figure 3A). However, starting the night period earlier or later did not lead to a concomitant shift in GA3ox1 induction (Figure 3B and C). This implies that GA3ox1 responds to darkness, but only when it occurs simultaneously with the expected onset of the night. The effect of carbon starvation on the nighttime GA3ox1 induction was investigated by DCMU and low light treatments, like done for daytime expressed $K S$ and GA20ox1. Although low light treatments led to an intermediate starvation response compared to DCMU (Supplemental Figure 2E), it was sufficient to completely abolish the nighttime induction of GA3ox1, identical to the effect of DCMU (Figure 3D). These observations suggest that GA3ox1 is more sensitive to carbon starvation. The relevance of energy and carbon signalling for GA3ox1 regulation was further investigated by transgenics, mutants and pharmaceutical inhibitors of three main energy signalling pathways, TARGET OF RAPAMYCIN (TOR) kinase, SNF1 RELATED PROTEIN KINASE 1 (SnRK1.1/KIN10) and glucose signalling (GLUCOSE INSENSITIVE2, GIN2)(22-24). Overexpression of the wild type alleles of neither KIN10 or TOR, nor the gin2-1 null allele affects the behaviour of GA3ox1 during night. However, preventing the activity of KIN10 and TOR kinase by either an active site specific mutation or pharmaceutical inhibitors, led to an enhanced or abolished nighttime GA3ox1 induction, respectively (Supplemental Figure 3). The direction of change corresponded to the removal of either a starvation, KIN10, or an energy abundance signal, TOR. 


\section{Bioactive gibberellins accumulate during the night and are severely reduced the morning after a day of low light levels.}

Patterns of transcriptional regulation suggest that gibberellin precursors are made during the day via the upstream enzyme $K S$ and pre-final enzyme GA200x1. These accumulated precursors would then be converted to bioactive GA by GA3ox1 which peaks during the night, leading to high bioactive GA the end of the night. Moreover, reduced light availability affected all three transcripts, especially GA3ox1 (Figure 2C and 3D). To investigate whether such transcriptional patterns would be translated into changes in the corresponding compounds, we measured gibberellin content at the start and end of the day. Moreover, we confronted plants with an extended day to reduce GA30x1 induction whilst maintaining carbon availability (Figure 3C).

Though the gibberellin precursor pool (GA9, GA19 and GA20) appeared higher in the evening, no significant differences were observed between timepoints nor between light levels (Figure 4A). The bioactive gibberellins differ in their affinity for the receptor GID1, with $G_{3}$ and $G_{1}$ having a weak affinity compared to strong binding affinity of $G A_{4}$ (16-17). The low affinity bioactive gibberellins $\left(G A_{1}\right.$ and $\left.G A_{3}\right)$ had no clear differences between treatments (Supplemental Figure 4). The high affinity gibberellin, $\mathrm{GA}_{4}$, was the most abundant bioactive gibberellin, and its levels were highest at the end of the night (Figure 4B). Reducing nighttime GA3ox1 induction by an extended day (Figure $3 C$ ), led to a subsequent drop in $\mathrm{GA}_{4}$ the following morning (Figure 4B). Low light levels led to an even stronger drop in $\mathrm{GA}_{4}$ the following morning, whereas $\mathrm{GA}_{4}$ levels remained unchanged at the end of the actual low light day (Figure 4B). These results suggest an important role of nighttime GA3ox1 expression in determining bioactive GA levels in response to low light, whereas the drop of $K S$ and GA20ox1 was not translated into reduced gibberellin biosynthesis within the time frame studied here. Gibberellin inactivating enzymes could also play a role in determining the dynamics of bioactive GA. However, no consistent changes of inactivated GAs $\left(\mathrm{GA}_{8}, \mathrm{GA}_{29}\right.$ and $\left.\mathrm{GA}_{34}\right)$ were found (Supplemental Figure 4).

Lower and higher levels of $\mathrm{GA}_{4}$ at the end of the day and night, respectively, suggest temporal dynamics in GA abundance. These direct gibberellin measurements are supported by in silico investigation of genes responsive to GA in Arabidopsis rosettes (25). For two independent datasets (20-21), the GA responsive genes were enriched among gene-sets that peaked in expression during the night, but underrepresented (depleted) among daytime peaking genes (Figure 4C, Suppl. Figure 5). These dynamics of gibberellin responsive genes could also be ascribed to changes in gibberellin sensitivity, as also observed in seedling hypocotyls (26). Indeed, transcript levels of GID1A seem to be highest during the evening and start of the night in rosettes (Supplemental Figure 1). The starchless mutant phosphoglucomutase (pgm), typically suffers from severe starvation at night $(21,27)$. Subsequently, the nighttime induction of GA3ox1 was abolished, whereas the daytime GA biosynthetic genes (KS and GA20ox1) remained mostly unaffected (Supplemental Figure 6A). Indeed, pgm and other starch mutants are susceptible to altered GA metabolism and reduced gibberellin levels (28). Subsequently, we found that rhythmic expression of GA responsive genes was lost in pgm, despite retaining rhythmicity in GID1A (Supplemental Figure 6B). The relevance of gibberellins in time of day specific leaf expansion was further investigated in the gibberellic acid insensitive (gai) and della pentuple (pent) mutants, which cannot respond to varying gibberellin levels (15). Expansion rates of gai and pent were decreased at night, when the expression of GA3ox1 determines the bioactive GA levels (Figure 5). Additionally. Expansion rates were elevated in the morning (Figure 5), which might represent a GA-independent behaviour that might utilize untapped nighttime growth potential. Likewise, a GA3ox1 knockout mutant, ga3ox1-3 (29), also has reduced nighttime expansion and elevated morning expansion (Figure 5). Overall, this suggests that an ability to respond to changes in gibberellin levels and GA biosynthesis contributes to time of day specific expansion.

\section{Reduced light availability leads to reduced leaf expansion rates during the following night only, which is partially mediated by GA3ox1.}

The next step was to investigate whether the carbon dependent regulation of GA3ox1 transcription and subsequent $\mathrm{GA}_{4}$ levels are important to adjust growth to periodic low light levels. To effectively investigate growth 
reductions, it is important that a mutant only has minor growth effects under control conditions (termed normal light), therefore a ga3ox1 knock down (SALK_025076) with a T-DNA insertion in the intron was used. This ga3ox1$k d$ had a more than 10 fold drop in GA3ox1-kd mRNA abundance and less than half the $\mathrm{GA}_{4}$ levels (Figure 6A, Supplemental Figure 7A-C). Furthermore, the low GA3ox1-kd mRNA abundance of the knock down was not significantly further reduced by a low light treatment (Figure 5B). The specific leaf area, an important growth parameter, was unaffected. However, ga3ox1-kd did flower slightly later and had reduced bolt length (Supplemental Figure 7D).

With the automated phenotyping platform WIWAM XY to precisely control soil water content and to image individual plants over time $(30,31)$ we followed the expansion rates of Col-0 and ga3ox1-kd plants under constant day/night cycles and when confronted with four interspersed low light days (Figure 6C). To obtain expansion rates under normal light and to determine low light mediated reductions in expansion we fitted growth curves to individual plants (Supplemental Figure 8). Under control conditions expansion rates were close to identical between Col-0 and ga3ox1-kd (Figure 6D). We concluded that GA3ox1-dependent fluctuations in gibberellin levels could not be the main driver of growth in ga3ox1 under normal light conditions. Exposure to the four low light days led to strongly reduced final rosette area in both genotypes (Supplemental Figure 8), implying that a few low light days can have dramatic effects. Quantification of the reduction in expansion during the low light day and subsequent night showed that ga3ox1-kd had a smaller reduction in expansion rate than Col-0 (Figure 6E). This suggests that expansion in ga3ox1-kd, in which the nighttime expression and regulation of GA3ox1 is negligible (Figure 6A-B), does not suffer as strongly from low light as the wild type, where low light severely dampens the expression of GA3ox1 (Figure 3D) and $\mathrm{GA}_{4}$ levels (Figure 4B).

To investigate the exact time of the day at which the growth reduction takes place and to further explore the role of GA3ox1 in such reductions, leaf expansion rates of the fastest growing leaf ( $7^{\text {th }}$ leaf; Supplemental Figure 9) was followed over 6- or 12-hour intervals. During the low light treatment, leaf expansion rates remained unchanged, whereas in the subsequent night, expansion was severely reduced (Figure 6F). Preventing a drop in $\mathrm{GA}_{4}$ after low light by either gibberellin application or the ga3ox1-kd, mitigated the reduction in expansion to a similar degree (Figure 6G). However, neither manipulation of gibberellins could completely abolish the growth penalty (Figure 6G). Similarly, for the full knock out of GA3ox1, ga3ox1-3, low light induced reduction of nighttime leaf expansion was mitigated (Figure 7H-I). Low light levels also lead to altered carbon dynamics in the subsequent day. The following day less carbon is allocated to structural biomass and subsequently soluble carbohydrates (including starch) accumulate to higher levels (32), and this accumulation the subsequent day was independent of gibberellins (Supplemental Figure 10A-B). The higher carbon availability coincided with enhanced growth during the second night after the low light treatment, especially in Col-0 where the low-light-induced expansion was of a similar magnitude as the expansion induced solely by exogenous gibberellins. The ga3ox1-kd seemed not able to benefit from this growth increase to the same extent (Supplemental Figure 10C).

\section{Discussion}

A capacity to adjust hormonal profiles to the prevailing environmental conditions is essential for plants to ensure optimal performance. For this reason, we aimed to identify how gibberellin metabolism was affected by carbon availability and the subsequent effects on growth. Nighttime production of $\mathrm{GA}_{4}$ by GA3ox1 was found to be highly sensitive to low light levels, which led to a reduction of $\mathrm{GA}_{4}$ levels only in the subsequent night and not the actual day. Similarly, effects on leaf expansion upon low light were only noticeable during the night and were mitigated in plants whose growth was not driven by nighttime expression of GA3ox1. The results presented here provide new insights into the regulation of gibberellin metabolism and add perspective on the mechanisms of growth control.

We found that reducing GA3ox1 mRNA levels, either through an extended day, low light availability or a genetic knockdown, consistently led to reduced $\mathrm{GA}_{4}$ abundance, but did not affect the levels of the low affinity bioactive 
gibberellins ( $\mathrm{GA}_{3}$ and $\mathrm{GA}_{1}$, Figure 4). Indeed, in vitro activity of $\mathrm{GA3}$ 30x1 was shown to have a strong preference for converting precursors to $\mathrm{GA}_{4}(33)$. Full knockouts of $\mathrm{GA3}$ ox 1 were also reported to have reduced $\mathrm{GA}_{4}$ levels $(29,34)$. In contrast, clearly reduced mRNA levels of upstream biosynthesis genes ( $K S$ and GA20ox1, Figure 2) did not lead to a corresponding reduction of precursors (Figure 4). GA20ox is generally considered the rate limiting step, since constitutive overexpression leads to higher $\mathrm{GA}_{4}$ levels, in contrast to overexpression of other biosynthesis genes (18). Longevity of prior produced enzymes could explain a lack of responses at the metabolite level observed after the short transcriptional perturbation of this study. Likely, successive days of reduced light availability would eventually also lead to an effect on precursor metabolism. However, $\mathrm{GA}_{4}$ levels were quick to respond to changes in GA3ox1, suggesting that GA3ox1 proteins need to be resynthesized daily. This implies that GA metabolism is reset every day via GA3ox1. The results presented here suggest that the abundance of GA3ox1 and GA20ox1 vary throughout the day-night cycle, and subsequently also the flux through each of these pathways.

It is tempting to attribute the low afternoon-growth and high night- and morning-growth (Figure $5 \mathrm{C}$ and $6 \mathrm{~F}$ ) with the corresponding changes in $\mathrm{GA}_{4}$ levels and behaviour of $\mathrm{GA}_{4}$ responsive genes (Figure 4). Gibberellin sensitivity was shown to be regulated by the circadian clock (26), here we show that also the actual levels of $\mathrm{GA}_{4}$ vary throughout the day and probably coincide with high gibberellin sensitivity (Figure 4, Supplemental Figure 1). Varying expansion rates of leaves throughout the day-night cycle are also regulated by carbon availability, water availability, light cues and the circadian clock $(6-8,31,35-36)$. It is not straight forward to piece apart the contributions of these growth cues and discern how they act in concert. Nevertheless, the results presented here show that light and dark cues, and the clock ensure timing of gibberellin biosynthesis and that disruption of biosynthesis patterns in the starchless pgm abolishes the rhythmic nature of downstream GA responses. Moreover, removing the ability to respond to varying gibberellin levels or disrupting biosynthesis impacts growth dynamics corresponding to the rhythmicity in GA biosynthesis.

The nighttime induction of GA3ox1 is highly dependent on carbon availability (Figure 3D). The consistently reduced nighttime growth penalty upon low light, either for whole rosettes or a single leaf, of plants without the capacity of GA3ox1 regulation demonstrates the relevance of this gene and subsequent gibberellins in adapting growth to the prevailing conditions (Figure 6). Although, the difference in the low light induced growth penalty between Col-0 and ga3ox1-kd is seemingly small, around 20-30\%, it is of a similar magnitude as a rescue by gibberellins (Figure 6E and G). A variety of growth stimulating gene regulatory networks have been identified in Arabidopsis and many of these act independent of one another (12-13). Likewise, the different plant hormones have surprisingly little overlap in downstream genes and appear to operate in distinct fashions (37). Alternative mechanisms that connect carbon and growth networks likely exist in plants (38). One is the starvation induced autophagy of brassinosteroid signalling components $(39,40)$. A collection of numerous of such growth regulating mechanisms will buffer against the loss of the single mechanism identified here, which could explain the small effect size. Similarly, boosting carbon assimilation by elevated $\mathrm{CO}_{2}$ can still enhance growth in paclobutrazol treated plants (41). In this study, plants were confronted by fewer photo-assimilates, which will place physical constraints on growth regardless of the manipulation of growth signalling mechanisms.

Gibberellins are crucial hormones for plants, which stimulate growth and development. This study reveals how gibberellin metabolism is balanced to match the physiology of the plant. We present a model of gibberellin biosynthesis that is coordinated throughout the day-night cycle by light cues, dark cues and the circadian clock. Here, the nighttime $\mathrm{GA}_{4}$ biosynthesis rate is reset daily and strongly depends on carbon availability. Starch, as the nighttime carbon source (42), plays a pivotal role in this model and acts as a robust integrator of daytime performance, unlike the highly variable nature of photosynthesis and light availability. Using starch, rather than photosynthesis directly, could prevent excessive starting and stopping of growth. Though the exact mechanism is unclear, plants can precisely estimate starch levels to ensure proper nighttime utilization rates $(42,43)$. Subsequently, starch utilization rates could be accurately used to pace gibberellin metabolism and aid in adjusting gibberellin responses, such as growth, to the prevailing conditions when plants grow in variable environments. 


\section{Methods}

Plant material, growth and treatments: Col-0 and ga3ox1 (NASC: N670439) were grown on a soil-perlite mixture. Light levels used for the experiments ranged from 100-130 $\mu \mathrm{mol} \mathrm{m}^{-2} \mathrm{~s}^{-1}$ photosynthetic active radiation (PAR) and relative humidity between 50 and $70 \%$ and a temperature of $20^{\circ} \mathrm{C}$. Low light treatments were between 30-45 $\mu \mathrm{mol} \mathrm{m} \mathrm{m}^{-2} \mathrm{~s}^{-1}$ PAR. DCMU $(100 \mu \mathrm{M})$ and $\mathrm{GA}_{4+7}(100 \mu \mathrm{M})$ were applied by spraying on the plants. Experiments were started once the plants had 11 visible leaves.

Gene expression and carbohydrates: For Gene expression two entire rosettes were pooled per replicate. RNA isolation was performed according to Kiefer method (40). DNA contaminant was removed with RQ1 RNase-Free DNase $^{\mathrm{TM}}$ (Promega) and cDNA was synthesized using Maxima First Strand cDNA Synthesis Kit ${ }^{\mathrm{TM}}$ (Thermofisher). The CDNA was used as template for real-time PCR (primers in Supplemental table 1) using CFV384 Touch ${ }^{\text {TM }}$ Real-Time PCR Detection System (Bio-Rad). Housekeeping genes (AT4G34270, AT1G13320) were selected based on ref. 45, with an extra focus on constants expression levels during the day-night cycle. Sugars and starch were determined through coupled enzymatic reactions and NADH absorbance at $340 \mathrm{~nm}$ as described previously (28).

Gibberellin metabolites Gibberellin levels were determined as previously described in ref. 46 with some modifications. In short, 2 to 4 grams of pooled shoot was homogenized in cold $80 \%(\mathrm{v} / \mathrm{v})$ methanol $(1: 5, \mathrm{w} / \mathrm{v})$ using a mortar and pestle. Fifty nanograms of deuterated GAs ([17,17-2H2]-GA9, [17,17-2H2]-GA4, [17,17-2H2]-GA34, [17,17-2H2]-GA19, [17,17-2H2]-GA20, [17,17-2H2]-GA29, [17,17-2H2]-GA1, [17,17-2H2]-GA8, [17,17-2H2]-GA3) were added as internal standards to account for purification losses. Methanol was evaporated under a vacuum at $35^{\circ} \mathrm{C}$, and the aqueous phase was partitioned against ethyl acetate, after adjusting the $\mathrm{pH}$ to 2.8 . The extracts were dried and suspended in $0.3-0.5 \mathrm{ml}$ of distilled water with $0.01 \%$ acetic acid and $10 \%$ methanol. HPLC analysis was performed with a Kontron instrument (Munich, Germany) equipped with a UV absorbance detector operating at $214 \mathrm{~nm}$. The samples were applied before to a $150 \times 4.6 \mathrm{~mm}$ ID column packed with ODS Hypersil (Thermo Fischer Scientific, Milan Italy), particle size $5 \mu \mathrm{m}$, eluted at a flow rate of $1 \mathrm{ml} \mathrm{min}{ }^{-1}$. The column was held constant at $10 \%$ methanol for $4 \mathrm{~min}$, followed by a double gradient elution from $10 \%$ to $30 \%$ and $30 \%$ to $100 \%$ over $40 \mathrm{~min}$. The fractions corresponding to the elution volumes of standard GAs were collected separately. Subsequently, the fractions were applied to $250 \times 4.6$ mm ID, Nucleosil 100-5 N(CH3)2 column (Macherey-Nagel GmbH \& Co, Düren, Germany) and eluted isocratically with $100 \%$ methanol containing $0.01 \%$ acetic acid at a flow rate of $1 \mathrm{ml} \mathrm{min}^{-1}$. The fractions corresponding to the elution volumes of standard GAs were collected, dried and silylated with $\mathrm{N}, \mathrm{O}$ bis (trimethylsilyl) trifluoroacetamide containing $1 \%$ trimethylchlorosilane (Pierce, Rockford, IL, USA) at $70{ }^{\circ} \mathrm{C}$ for 1-h. The gas chromatography-tandem mass spectrometry analysis was performed on a Saturn 2200 quadruple ion trap mass spectrometer coupled to a CP-3800 gas chromatograph (Varian Analytical Instruments) equipped with a MEGA (http://www.mega.mi.it) 1MS capillary column (30 m $30.25-\mathrm{mm}$ i.d. and $0.25-\mu \mathrm{m}$ film thickness). The carrier gas was helium, which was dried and air free, with a linear speed of $60 \mathrm{~cm} \mathrm{~s}^{-1}$. The oven temperature was maintained at $80^{\circ} \mathrm{C}$ for $2 \mathrm{~min}$ and increased to $300^{\circ} \mathrm{C}$ at a rate of $10^{\circ} \mathrm{C} \mathrm{min}{ }^{-1}$. Injector and transfer line were set at $250^{\circ} \mathrm{C}$ and the ion source temperature at $200^{\circ} \mathrm{C}$. Full scan mass spectra were obtained in El+ mode with an emission current of $10 \mu \mathrm{A}$ and an axial modulation of $4 \mathrm{~V}$. Data acquisition was from 150 to $650 \mathrm{Da}$ at a speed of 1.4 scan s$^{-1}$. GAs were identified by comparing the full mass spectra with those of the authentic compounds, and quantification with reference to standard plots of concentration ratios versus ion ratios that were obtained by analysing known mixtures of unlabelled and labelled GAs.

Growth measurements: The Weighing, Imaging and Watering Automated Machine (WIWAM) (www.wiwam.com) to precisely control soil water content and image plants was used to follow size increase of individuals over time. The PSB Interface for Plant Phenotype Analysis (PIPPA, https://pippa.psb.ugent.be) was used for analysis, visualization and management of phenotypic datasets and images. A four-parameter logistic model (constant conditions) or a series of exponential models (low light treatment) were fitted to individual plants. This allowed us to track growth rates over time and rosette area. Contributions of distinct leaves to total size increase was 
determined by two destructive harvests, separated by 24 hours. Leaves were dissected and arranged on $0.59 \%$ agarose filled plates, leaf incisions ensured proper flattening of the leaves. Areas were determined with ImageJ software on pictures obtained by scanning the plates. Growth of individual leaves over time were determined, non-destructively, by manual length measurements with a digital calliper.

In silico analysis and statistics. Data analysis and statistics were done using R software. Data were transformed if needed to satisfy the assumptions of statistical tests. Calling of rhythmic genes in pgm was done by the HAYSTACK tool from the DIURNAL project (47).

\section{Acknowledgments}

This work was supported by the Scuola Superiore Sant' Anna (SSSA) and the Plantecophysiology group (Utrecht University). P.Pr was supported by a PhD fellowship in Agrobiodiversity (SSSA). P.Pe. and L.M. acknowledge support of CISUP at University of Pisa. S.D. was funded by Research Foundation Flanders (FWO) by a Postdoctoral Fellowship. H.v.V was supported by SSSA and grant ALWOP.419 from the dutch scientific organisation. We further acknowledge financial support by the Access to Research Infrastructures activity in the Horizon2020 Programme of the EU (EPPN2020 Grant Agreement 731013).

\section{References}

1. H. Nelissen, N. Gonzalez, D. Inzé, Leaf growth in dicots and monocots: so different yet so alike. Current opinion in plant biology, 33, $72-76$ (2016)

2. R. Sulpice, A.F. Alexander, A. Ivakov, F. Apelt, N. Krohn, B. Encke, C. Abel, R. Feil, J.E. Lunn, M. Stitt. Arabidopsis Coordinates the Diurnal Regulation of Carbon Allocation and Growth across a Wide Range of Photoperiods. Molecular Plant, 7(1), 137-155, (2014)

3. V. Mengin, E-T. Pyl, T.A. Moraes, R. Sulpice, N. Krohn, B. Encke, M. Stitt. Photosynthate partitioning to starch in Arabidopsis thaliana is insensitive to light intensity but sensitive to photoperiod due to a restriction on growth in the light in short photoperiods. Plant, Cell \& Environment, 40, 2608- 2627 (2017)

4. S.K. Pal, M. Liput, M. Piques, H.Ishihara, T. Obata, M.C.M. Martins, R. Sulpice, J.T. van Dongen, A.R. Fernie, U.P. Yadav, J.E. Lunn, B. Usadel, M. Stitt. Diurnal Changes of Polysome Loading Track Sucrose Content in the Rosette of Wild-Type Arabidopsis and the Starchless pgm Mutant Plant Physiology 162 (3) 1246-1265 (2013)

5. Ikakov, A. Flis, F. Apelt, M. Fünfgeld, U. Scherer, M. Stitt, F. Kragler, K. Vissenberg, S. Persson, D. Suslov. Cellulose synthesis and cell expansion are regulated by different mechanisms in growing Arabidopsis hypocotyls. The Plant Cell 29(6) 1305-1315 (2017)

6. Farré, E. M. The regulation of plant growth by the circadian clock. Plant Biology, 14: 401-410. (2012)

7. T. Dornbusch, O. Michaud, I. Xenarios, C. Fankhauser. Differentially phased leaf growth and movements in Arabidopsis depend on coordinated circadian and light regulation. The Plant Cell 26 (10), 3911-3921 (2014)

8. F. Apelt, D. Breuer, J.J. Olas, M.G. Annunziata, A. Flis, Z. Nikoloski, F. Kragler, M. Stitt. Circadian, Carbon, and Light Control of Expansion Growth and Leaf Movement. Plant Physiology, 174 (3) 1949-1968 (2017)

9. N. Olszewski, T.P Sun, F. Gubler. Gibberellin signaling: biosynthesis, catabolism, and response pathways. The Plant Cell, 14 S61S80. (2002)

10. P. Achard, A. Gusti, S. Cheminant, M. Alioua, S. Dhondt, F. Coppens, G.T.S. Beemster, P. Genschik. Gibberellin Signaling Controls Cell Proliferation Rate in Arabidopsis. Current Biology 19(14) 1188-1193 (2009)

11. P. Hedden, S.G. Thomas. Gibberellin biosynthesis and its regulation. Biochem J. 15;444(1):11-25 (2012)

12. N. Gonzalez, S. De Bodt, R. Sulpice, Y. Jikumaru, E. Chae, S. Dhondt, T. Van Daele, L. De Milde, D. Weigel, Y. Kamiya, M. Stitt, G.T.S. Beemster, D. Inzé. Increased Leaf Size: Different Means to an End. Plant Physiology, 153 (3) 1261-1279 (2010)

13. H. Vanhaeren, N. Gonzalez, F. Coppens, L. De Milde, T. Van Daele, M. Vermeersch, N.B. Eloy, V. Storme, Dirk Inzé. Combining growth-promoting genes leads to positive epistasis in Arabidopsis thaliana. eLife 2014;3:e02252 (2014)

14. Y.J. Nam, D. Herman, J. Blomme, E. Chae, M. Kojima, F. Coppens, V. Storme, T. Van Daele, S. Dhondt, H. Sakakibara, D. Weigel, D Inzé, N. Gonzalez. Natural Variation of Molecular and Morphological Gibberellin Responses. Plant Physiology 173(1):703-714 (2017)

15. N. Pullen, N. Zhang, A.D. Alonso, S. Penfield. Growth rate regulation is associated with developmental modification of source efficiency. Nature Plants 5, 148-152 (2019)

16. M. Ueguchi-Tanaka, M. Ashikari, M Nakajima, H Itoh, E. Katoh, M. Kobayashi, T.Y. Chow, Y.I.C. Hsing, H. Kitano, I. I. Yamaguchi, M. Matsuoka. GIBBERELLIN INSENSITIVE DWARF1 encodes a soluble receptor for gibberellin. Nature 437: 693-698. (2005) 

made available under aCC-BY-NC 4.0 International license.

17. H. Yoshida, E. Tanimoto, T. Hirai, Y. Miyanoiri, R. Mitani, M. Kawamura, M. Takeda, S. Takehara, K. Hirano, M. Kainosho, T. Akagi, M. Matsuoka, M. Ueguchi-Tanaka . Evolution and diversification of the plant gibberellin receptor GID1. Proceedings of the National Academy of Sciences, 115(33), E7844-E7853. (2018)

18. S. Yamaguchi. Gibberellin metabolism and its regulation. Annu. Rev. Plant Biol. 59, 225-251 (2008)

19. A.M. Middleton, S. Úbeda-Tomás, J. Griffiths, T. Holman, P. Hedden, S.G. Thomas, A.L. Phillips, M.J. Holdsworth, M.J. Bennett, J.R. King, M.R. Owen. Mathematical modeling elucidates the role of transcriptional feedback in gibberellin signaling. PNAS 109 (19) 7571-7576 (2012)

20. S.M Smith, D.C. Fulton, T. Chia, D. Thorneycroft, A. Chapple, H. Dunstan, C. Hylton, S.C. Zeeman, A.M. Smith. Diurnal Changes in the Transcriptome Encoding Enzymes of Starch Metabolism Provide Evidence for Both Transcriptional and Posttranscriptional Regulation of Starch Metabolism in Arabidopsis Leaves. Plant Physiology 136: 2687-2699. (2004)

21. O.E. Bläsing, Y. Gibon, M. Günther, M. Höhne, R. Morcuende, D. Osuna, O. Thimm, B. Usadel, W.R. Scheible, M. Stitt. Sugars and circadian regulation make major contributions to the global regulation of diurnal gene expression in Arabidopsis. The Plant Cell, 17(12), 3257-3281. (2005)

22. E. Baena-González, F.Rolland, J.M. Thevelein, J. Sheen. A central integrator of transcription networks in plant stress and energy signalling. Nature 448, 938-942 (2007).

23. D. Deprost, L. Yao, R. Sormani, M. Moreau, G. Leterreux, M. Nicolaï, M. Bedu, C. Robaglia, C. Meyer. The Arabidopsis TOR kinase links plant growth, yield, stress resistance and mRNA translation EMBO Reports 8:864-870 (2007)

24. B., Moore, L. Zhou, F. Rolland, Q. Hall, W.H. Cheng, Y.X. Liu, I. Hwang, T. Jones, J. Sheen. Role of the Arabidopsis glucose sensor HXK1 in nutrient, light, and hormonal signaling. Science, 300(5617):.332-336. (2003)

25. D.M. Ribeiro, W.L. Araújo, A.R. Fernie, J.H.M Schippers, B. Mueller-Roeber. Translatome and metabolome effects triggered by gibberellins during rosette growth in Arabidopsis. Journal of Experimental Botany 63: 2769-2786. (2012)

26. M.V. Arana, N. Marín-de la Rosa, J.N. Maloof, M.A. Blázquez, D. Alabadí. Circadian oscillation of gibberellin signaling in Arabidopsis. Proceedings of the National Academy of Sciences, 108(22), 9292-9297. (2011)

27. T. Caspar, S.C. Huber, C. Somerville. Alterations in growth, photosynthesis, and respiration in a starchless mutant of Arabidopsis thaliana (L.) deficient in chloroplast phosphoglucomutase activity. Plant Physiology, 79(1), 11-17 (1985)

28. E. Paparelli, S. Parlanti, S. Gonzali, G. Novi, L. Mariotti, N. Ceccarelli, J.T. van Dongen, K. Kölling, S.C. Zeeman, P. Perata Nighttime sugar starvation orchestrates gibberellin biosynthesis and plant growth in Arabidopsis. The Plant Cell, 25: 3760-3769 (2013)

29. M.G. Mitchum, S. Yamaguchi, A. Hanada, A. Kuwahara, Y. Yoshioka, T. Kato, S. Tabata, Y. Kamiya, T.P. Sun. Distinct and overlapping roles of two gibberellin 3-oxidases in Arabidopsis development. The Plant Journal. 45(5):804-18. (2006)

30. P. Clauw,F Coppens, K. De Beuf, S Dhondt, T. Van Daele, K. Maleux, V. Storme, L. Clement, N. Gonzalez, D. Inzé. Leaf responses to mild drought stress in natural variants of Arabidopsis. Plant Physiology 167:800-16 (2015)

31. M. Dubois, H. Claeys, L. Van den Broeck, D. Inzé. Time of day determines Arabidopsis transcriptome and growth dynamics under mild drought. Plant, Cell \& Environment, 40: 180- 189 (2017)

32. T.A. Moraes, V. Mengin, M.G. Annunziata, B. Encke, N. Krohn, M. Hoehne, M. Stitt. Response of the circadian clock and diel starch turnover to one day of low light or low $\mathrm{CO}_{2}$. Plant physiology, 179(4), 1457-1478. (2019)

33. J. Williams, A.L. Phillips, P. Gaskin, P. Hedden. Function and Substrate Specificity of the Gibberellin $3 \beta$-Hydroxylase Encoded by the Arabidopsis GA4 Gene. Plant Physiology, 117 (2) 559-563 (1998)

34. J. Hu, M.G. Mitchum, N. Barnaby, B.T. Ayele, M. Ogawa, E. Nam, W.C. Lai, A. Hanada, J.M. Alonso, J.R. Ecker JR, S.M. Swain, S. Yamaguchi, Y. Kamiya, T.-P. Sun. Potential sites of bioactive gibberellin production during reproductive growth in Arabidopsis. The Plant Cell 20(2):320-36. (2008)

35. K. Nozue, J.N. Maloof. Diurnal regulation of plant growth Plant, Cell \& Environment, 9 (3) 396-408 (2006)

36. F. Pantin, T. Simonneau, G. Rolland, M. Dauzat, B. Muller. Control of leaf expansion: a developmental switch from metabolics to hydraulics. Plant Physiology 156, 803-815. (2011)

37. J.L. Nemhauser, H. Fangxin, J. Chory. Different Plant Hormones Regulate Similar Processes through Largely Nonoverlapping Transcriptional Responses. Cell 126(3), 467-475 (2006)

38. K. Ljung, J.L. Nemhauser, P. Perata. New mechanistic links between sugar and hormone signalling networks. Current Opinions in Plant Biology 25, 130-137 (2015)

39. Z. Zhang, J.-Y. Zhu, J. Roh, C. Marchive, S.-K. Kim, C. Meyer, Y. Sun, W. Wang, Z.-Y. Wang. TOR Signaling Promotes Accumulation of BZR1 to Balance Growth with Carbon Availability in Arabidopsis. Current Biology 26(14) 1854-1860 (2016)

40. T.M. Nolan, B. Brennan, M. Yang, J. Chen, M. Zhang, Z. Li, X. Wang, D.C.Bassham, J. Walley, Y. Yin. Selective Autophagy of BES1 Mediated by DSK2 Balances Plant Growth and Survival. Developmental Cell, 41(1) 33-46.e7 (2017)

41. D.M. Ribeiro, W.L. Araújo, A.R. Fernie, J.H.M. Schippers, B. Mueller-Roeber. Action of Gibberellins on Growth and Metabolism of Arabidopsis Plants Associated with High Concentration of Carbon Dioxide. Plant Physiology 160(4): 1781-1794 (2012)

42. A. Graf, A. Schlereth, M. Stitt, A.M. Smith. Circadian control of carbohydrate availability for growth in Arabidopsis plants at night. PNAS 107 (20), 9458-9463 (2010)

43. A. Scialdone, S.T. Mugford, D. Feike, A. Skeffington, P. Borrill, A. Graf, A.M. Smith, M. Howard. Arabidopsis plants perform arithmetic division to prevent starvation at night. eLife 2:e00669 (2013) 
bioRxiv preprint doi: https://doi.org/10.1101/2020 05.06.080358; this version posted May 7, 2020. The copyright holder for this preprint (which was not certified by peer review) is the author/funder, who has granted bioRxiv a license to display the preprint in perpetuity. It is made available under aCC-BY-NC 4.0 International license.

44. E. Kiefer, W. Heller, D. Ernst. A simple and efficient protocol for isolation of functional RNA from plant tissues rich in secondary metabolites. Plant Molecular Biology Reporter 18: 33-39. (2000)

45. T. Czechowski, M. Stitt, T. Altmann, M.K. Udvardi, W.R. Scheible. Genome-wide identification and testing of superior reference genes for transcript normalization in Arabidopsis. Plant physiology, 139(1) 5-17. (2005)

46. L. Mariotti, P Picciarelli, L Lombardi, N Ceccarelli. Fruit-set and Early Fruit Growth in Tomato are Associated with Increases in Indoleacetic Acid, Cytokinin, and Bioactive Gibberellin Contents. Journal of Plant Growth Regulation 30: 405-415. (2011)

47. T.C. Mockler, T.P. Michael, H.D. Priest, R. Shen, C.M. Sullivan, S.A. Givan, C. McEntee, S.A. Kay, J. Chory. The DIURNAL project: DIURNAL and circadian expression profiling, model-based pattern matching, and promoter analysis. In Cold Spring Harbor symposia on quantitative biology (Vol. 72, pp. 353-363). Cold Spring Harbor Laboratory Press. (2007) 
bioRxiv preprint doi: https://doi.org/10.1101/2020.05 06.080358; this version posted May 7, 2020. The copyright holder for this preprint (which was not certified by peer review) is the author/funder, who has granted bioRxiv a license to display the preprint in perpetuity. It is made available under aCC-BY-NC 4.0 International license.

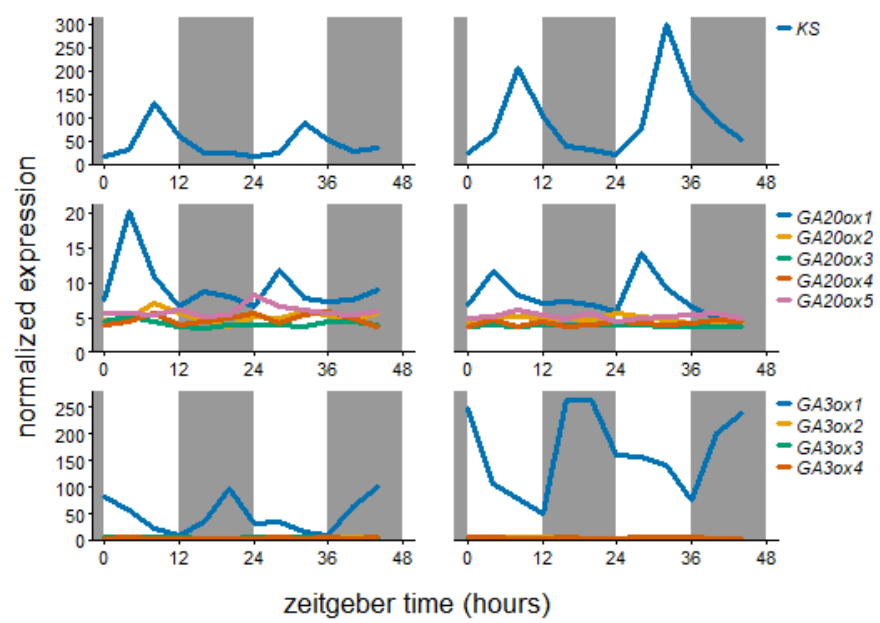

Figure 1. mRNA abundance over the day-night cycle of the gene family members of rhythmic GA biosynthetic genes.

Left and right column based on data from 35 day and 29 day old, respectively, soil grown Col-0 rosettes (20-21). Grey boxes represent the night period. All family members are shown. 

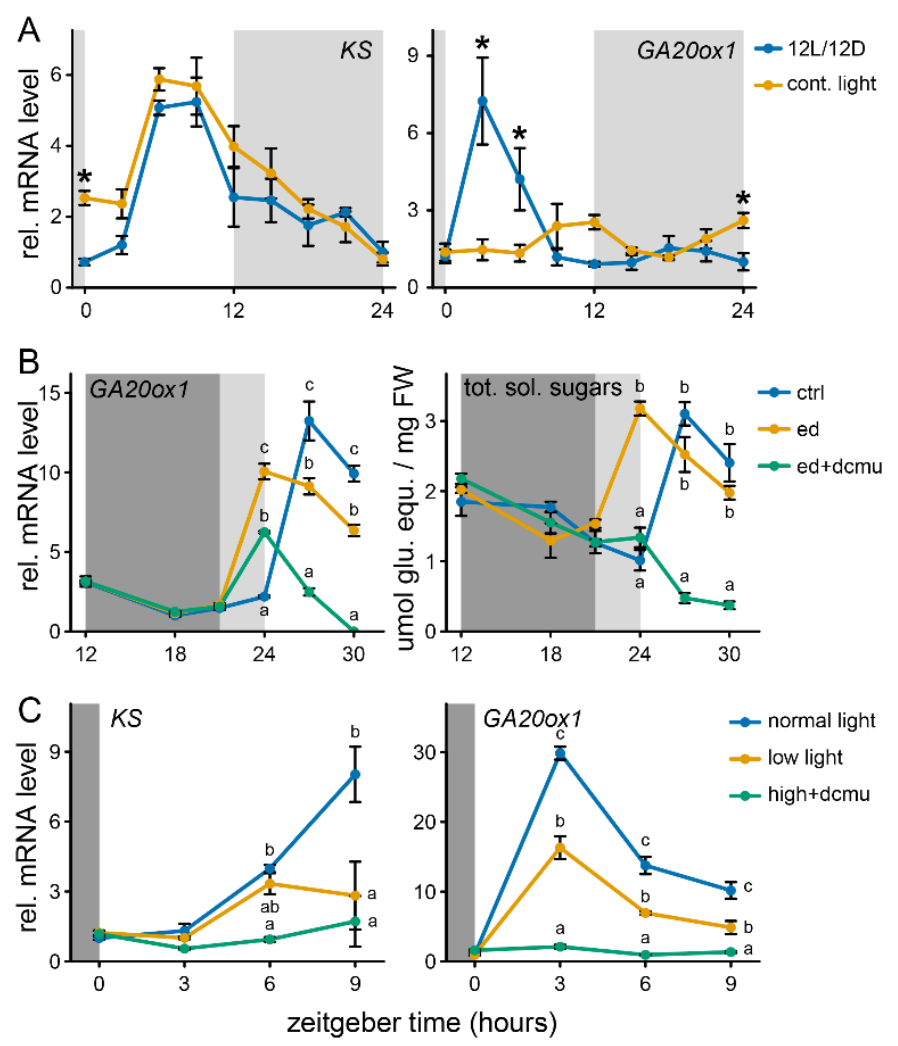

Figure 2. Transcriptional regulation of the daytime peaking GA biosynthetic genes, KS and GA200x1.

A. The role of the circadian clock in mediating rhythmic expression. Light grey boxes represent subjective night. Sampling started the second day after beginning continuous light conditions. B. Effect of a 3 hour early start of day. Dark and light grey represent night and the subjective night, respectively. DCMU and mock solution $(100 \mu \mathrm{M})$ was applied at ZT12. Ctrl: 12L/12D, ed: early start of day. C. The effect of reduced light availability or DCMU on KS and GA20ox1 transcript abundance. DCMU was applied at the start of the preceding night. All data are from 10 leaf stage soil grown Col-0 rosette. Mean \pm sem are shown, $n=4$. Asterisks (planned comparisons) and letters (Tukey HSD) represent statistically significant difference at specific time points $p<0.05$ ) 


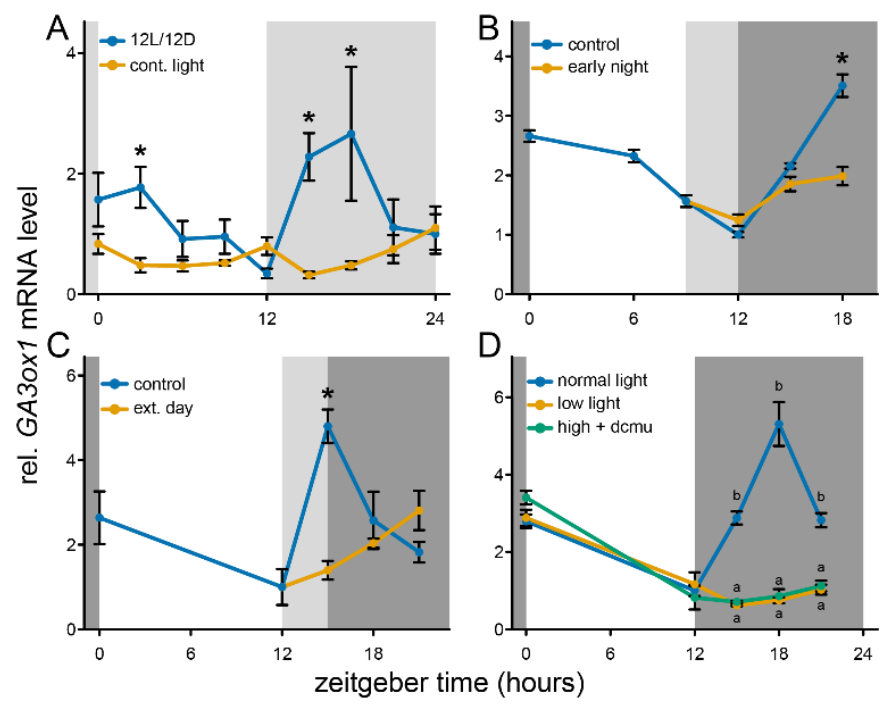

Figure 3. Transcriptional regulation of the night-time peaking GA biosynthetic gene, GA3ox1.

A. The role of the circadian clock in mediating rhythmic expression. Light grey boxes represent subjective night. Sampling started second day after starting continuous light conditions. B. Effect of a 3 hour early start of the night. Dark and light grey represent night and the subjective night, respectively. C. Effect of a 3 hour extension of the day. Dark and light grey represent night and the subjective night, respectively. D. The effect of reduced light availability or DCMU on nighttime GA3ox1 transcript abundance. DCMU was applied at the start of the preceding night. All data are from $\sim 10$ leaf stage soil grown Col- 0 rosette. Mean \pm sem are shown, $n=4$. Asterisks (planned comparisons) and letters (Tukey HSD) represent statistically significant difference at specific time points $p<0.05$ ) 

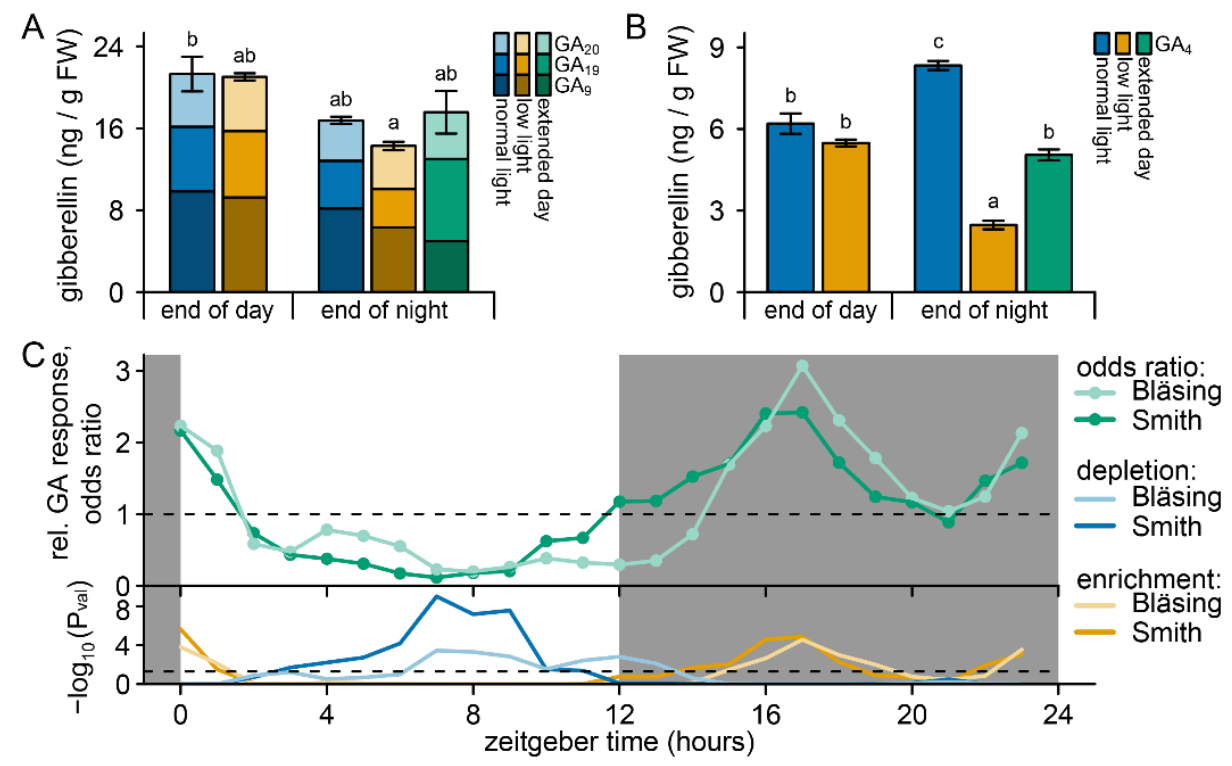

Figure 4. GA abundance and responses over time and after low light conditions

A. Abundance of gibberellin precursors metabolized by GA20ox, directly following a control (high light) or low light day (end of day, ZT12) and after the following subsequent night (end of night, ZT24). Extended day (under control light conditions) represents a 3 hour delay in the start of the night as in Figure $2 \mathrm{C}$. Data are mean, sem of the sum of precursors is shown. Letters indicate significant differences $(P<0.05$, Tukey HSD, $N=3)$. B. Conditions as in $A$, but levels of the main bioactive $\mathrm{GA}_{4}$ are shown. C. Relative enrichment (odd score $>1$ ) or depletion (odd score $<$ 1) of GA responsive genes among gene-sets that have rhythmic behaviour peaking at specific times of the day. Gene sets for each time of day were obtained from $(20-21,47)$. GA responsive genes were based on GA treated rosettes (22). Below is the statistical significance of depletion or enrichment of GA responsive genes (Hypergeometric distribution). Dashed line indicates $\mathrm{P}=0.05$. 


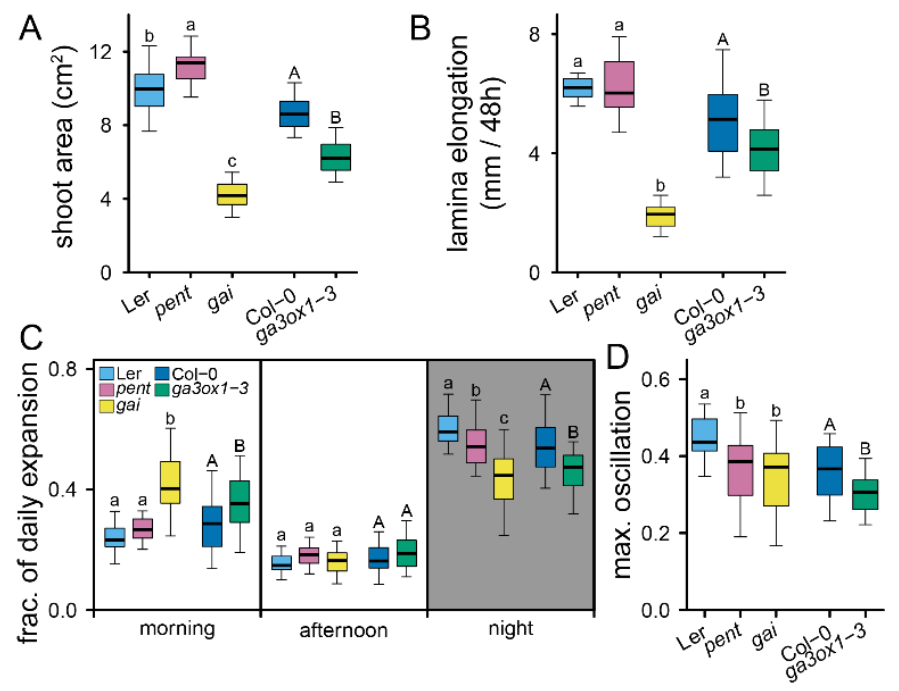

Figure 5. Daily changes in expansion

A. Projected shoot area 3 weeks after transplanting. B. Lamina expansion of leaf 7 in a 10 leaf rosette over 48 hours. C. portion of the daily expansion occurring during the morning (ZT0 - ZT6), afternoon (ZT6-ZT12) and night (ZT12-ZT24). The average of two days is shown. D. The extent of growth rhythmicity expressed as the maximum difference in fraction of daily expansion between the three time periods investigated (morning, afternoon and night) growth rate difference. Letters indicate significant differences $(P<0.05$, Tukey HSD, $N=25)$, lower and uppercase distinguish comparisons for the two different genetic backgrounds used. Pent = della pentuple mutant. 

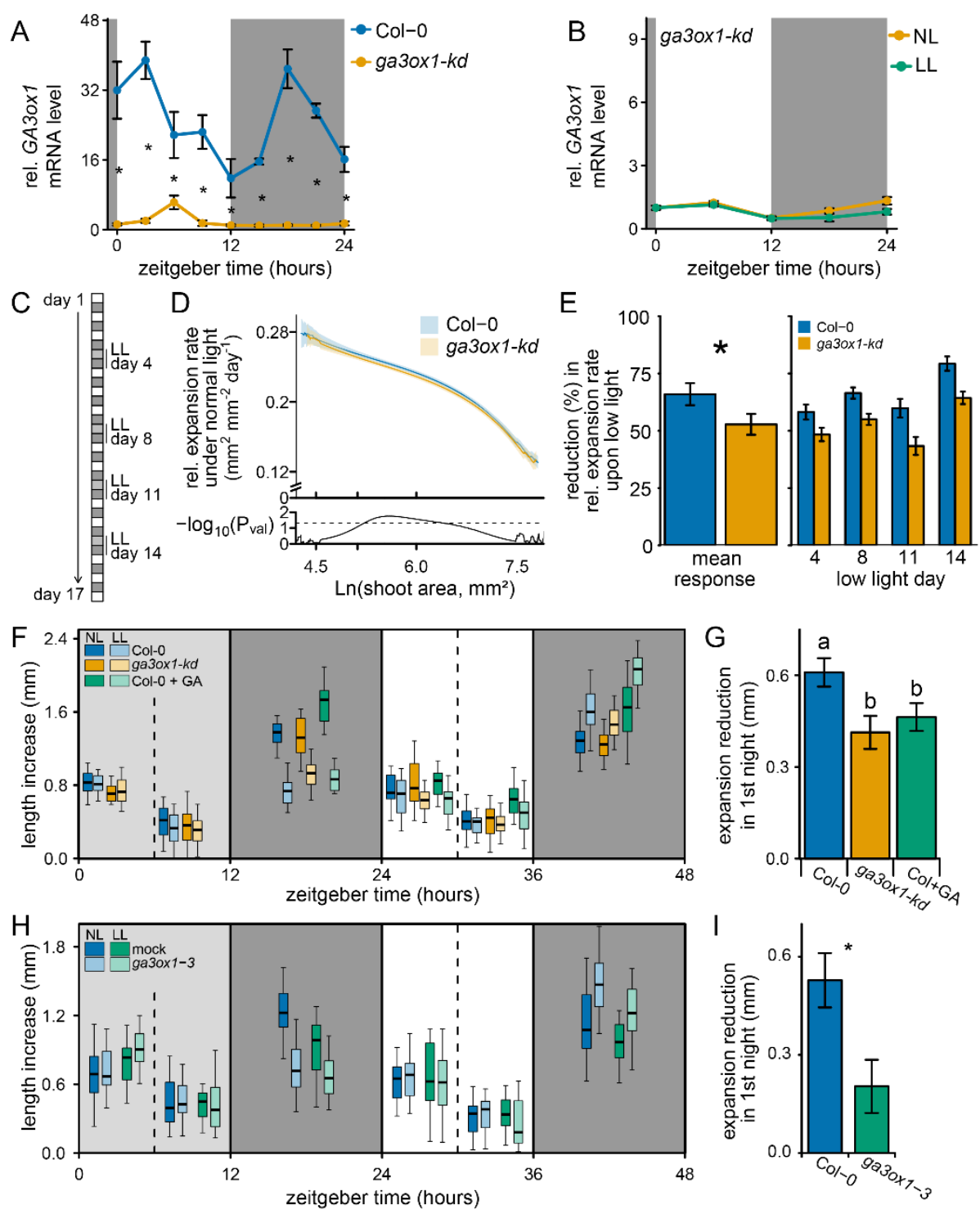

Figure 6. Carbon and GA dependent growth under constant and variable conditions

A. Transcript levels of GA3ox 1 in Col-0 and the ga3ox1-kd line, mean \pm sem $(\mathrm{N}=4)$. B. The behaviour of GA3ox1 during low light (LL) and normal light (NL) conditions in ga3ox1-kd, mean \pm sem (N=5). C. A brief schematic of the measuring and treatment regime. D. Expansion rate during constant high light conditions for a given projected shoot area. The shaded areas indicate the $95 \%$ confidence interval of the mean. The statistical difference between genotypes (students t-test) is given below $(\mathrm{N}=40)$. Dashed line indicates $\mathrm{P}=0.05$. Based on control plants $(\mathrm{NL})$ not confronted by low light days. E. The reduction in relative expansion rate during a low light day and its subsequent night compared to the expansion rate of the previous days. The reduction for each individual low light day, and the mean low light response over all 4 days are shown. Asterisks indicate the significant difference (ANOVA), mean \pm sem $(\mathrm{N}=40)$. F. Lamina length increase (leaf 7$)$ over measured time intervals (6 or 12 hours), during and following a low light day. Dark and light grey boxes indicate the night and the low light treatment respectively. Gibberellin $\left(\mathrm{GA}_{4+7}\right)$ /mock was applied at ZT12. N=25, 10 leaf stage plants. G. Reduction in expansion compared to normal light plants specifically at the night following a low light treatment (ZT12 to ZT24), mean \pm sem (N=25). Low light Col+GA growth reduction is compared to Col-0 normal light + mock. Letters indicate significant differences $(P<0.05$, Tukey HSD). $\mathbf{H}$ and $\mathbf{l}$. Experiment identical to $\mathrm{F}$ and $\mathrm{G}$, but with Col-0 and the full knock out ga3ox1-3. Mean \pm sem $(\mathrm{N}=25)$ is shown, asterisk indicates statistical significance. NL: control normal light conditions, LL: low light conditions. 